\title{
Reporte del primer hallazgo de puma (Puma concolor puma) infectado con Trichinella sp. en Chile ${ }^{\#}$
}

\author{
Report of the first finding of puma (Puma concolor puma) infected with Trichinella sp. in Chile \\ A Hidalgo ${ }^{\mathrm{a}}$, CA Oberg ${ }^{\mathrm{b}, \mathrm{c}}$, F Fonseca-Salamanca ${ }^{\mathrm{b}, \mathrm{c}^{\mathrm{k}}}$, MF Vidal ${ }^{\mathrm{a}, \mathrm{d}}$ \\ ${ }^{a}$ Escuela de Medicina Veterinaria, Universidad Santo Tomás, Temuco, Chile. \\ ${ }^{\mathrm{b}}$ Departamento de Ciencias Preclínicas, Unidad de Parasitología, Universidad de La Frontera, Temuco, Chile. \\ ${ }^{c}$ Laboratorio de Inmunoparasitología Molecular, Centro de Excelencia en Estudios Genéticos e Inmunológicos (CEGIN), \\ Universidad de La Frontera, Temuco, Chile. \\ ${ }^{\mathrm{d}}$ Fundación Fauna Andina Los Canelos, Villarrica, Chile.
}

\begin{abstract}
SUMMARY
Currently, Trichinella spiralis is the only species that has been identified in Chile as an agent of trichinellosis in both domestic and wild hosts. Preliminary studies have not identified the infection in animals native to Chile. We report the first finding of Trichinella sp. isolated in diaphragmatic and intercostal muscle of a puma (Puma concolor puma) found dead by telemetry tracking and subjected to necropsy on the mountain range of Región de La Araucanía, Chile. The diagnosis was made by trichinoscopy and artificial digestion. This finding demonstrates the presence of Trichinella sp. in chilean wild carnivorous animals.
\end{abstract}

Keywords: Trichinella, Puma concolor, zoonosis.

\section{RESUMEN}

En la actualidad, Trichinella spiralis es la única especie que ha sido identificada en Chile como agente de la trichinellosis, tanto en hospedadores domésticos como silvestres. Estudios preliminares no han identificado aún la infección por Trichinella en animales nativos de Chile. La presente comunicación, informa del primer hallazgo de Trichinella sp. aislado en musculatura diafragmática e intercostales de un ejemplar de Puma (Puma concolor puma) encontrado muerto mediante seguimiento por telemetría en el sector cordillerano de la Región de La Araucanía, Chile. El diagnóstico se realizó mediante triquinoscopía y digestión artificial. Este hallazgo confirma la existencia de Trichinella sp. en carnívoros nativos de Chile.

Palabras clave: Trichinella, Puma concolor, zoonosis.

\section{INTRODUCCIÓN}

Trichinella sp. es un nematodo que habita el intestino delgado de una amplia variedad de mamíferos, los estados larvales forman quistes en el tejido muscular, transmitiéndose por carnivorismo. El hombre se infecta por el consumo de tejido muscular de huéspedes infectados (Acha y Szyfres 2001).

En Chile, esta zoonosis es clasificada por el Ministerio de Salud, como enfermedad de notificación obligatoria diaria, siendo el consumo de cerdo la principal fuente de infección humana. Es considerada endémica en el país y ha sido descrita en cerdos (Plaza y col 1961, Sanhueza y Álvarez 1980, Alcaíno y col 1981), perros y gatos (Lardiez 1964, Letonja y Ernst 1974, Oberg y col 1979) y ratas (Neghme y col 1948, Rojas y col 1971, Schenone y col 1967 a,b).

Aceptado: 27.12.2012.

\# Financiado por la Universidad de La Frontera, Proyecto DIUFRO $\mathrm{N}^{\mathrm{o}}$ DI08-0014

* Casilla 54 D, Temuco, Chile; flery.fonseca@ufrontera.cl
En los últimos 15 años, los estudios sobre su taxonomía y hospedadores se han incrementado notablemente, describiéndose actualmente en el género Trichinella un cluster dividido en dos grupos: especies encapsuladas y no encapsuladas, con un total de diez especies y cuatro a nivel de genotipo (Murrell y col 2000, La Rosa y col 2003, Pozio 2009), siendo Trichinella T12 un genotipo descrito recientemente en la Patagonia Argentina (Krivokapich y col 2008).

La identificación de nuevos genotipos o cepas propias de Sudamérica como T12, podría explicar la infección por Trichinella sp. en "El Niño del Cerro El Plomo", momia incásica del 1500 D.C., hallazgo que cambiaría la teoría de que el parásito fue introducido con los cerdos y ratas durante la colonización europea (Rodríguez y col 2011).

Los escasos estudios moleculares realizados mediante PCR han identificado a la especie T. spiralis como la especie presente en Chile (Schenone y col 2002, Fonseca-Salamanca y col 2006), confirmando la ocurrencia del ciclo doméstico, el cual en otras zonas endémicas podría 
propagarse a animales silvestres a través de especies sinantrópicos como ratas, gatos, perros y otros, favoreciendo el flujo de $T$. spiralis hacia el medio silvestre. De la misma forma, genotipos silvestres podrían ser traspasados hacia el ciclo doméstico (Martínez 1999), referente a esto, Pozio (2000) reconoce un ciclo sinantrópico entre el doméstico y el silvestre.

Estudios realizados entre 1957 y 1962 en 5 especies de carnívoros (301 ejemplares), 9 especies de roedores (1.762 animales) y 80 ballenas cazadas en aguas chilenas, fueron negativos a la presencia de Trichinella sp. en animales silvestres nativos (Alcaíno y Arenas 1981). Los registros más cercanos provienen de Argentina, donde la infección ha sido diagnosticada en especies nativas como armadillo y puma (Neghme y Schenone 1970, Ribicich y col 2010).

Puma concolor, Linnaeus 1771, es un mamífero ampliamente distribuido en el cono Sur de América y habita una amplia diversidad de ambientes (Wilson y Reeder, 2005). En Chile ha sido identificada la subespecie P. concolor puma (Molina, 1782), siendo el carnívoro terrestre de mayor tamaño y de más amplia distribución geográfica (Quintana y col 2000). Es especie protegida clasificada como vulnerable o en peligro, cuya captura, caza y comercialización está prohibida desde los inicios de 1980 por el Decreto 354 del Servicio Agrícola y Ganadero (SAG). A pesar de ello, es cazado ilegalmente, consumido ocasionalmente y representa un riesgo de salud pública como una posible fuente de infección de triquinelosis humana. Así lo demuestra Mansilla y col (1994), quienes documentaron dos casos producidos por consumo de carne de puma en el sur de Argentina.

El presente artículo tiene como objetivo informar el primer hallazgo microscópico de Trichinella sp. en Puma concolor puma en la Región de La Araucanía, Chile.

\section{MATERIAL Y MÉTODOS}

En agosto de 2008, en el marco del proyecto Puma Araucano de Fundación Fauna Andina Los Canelos, fue recuperado un cadáver de Puma concolor puma, procedente de Nueva Imperial, Región de La Araucanía (lat.$38^{\circ} .73$ long. $-72^{\circ} .95$ ) el cual era monitoreado mediante radio collar y telemetría, de sexo hembra, de 5 años, cuya edad fue determinada según la cronología dentaria propuesta por Laundre y col (2000) y Shaw y col (2007). El ejemplar fue sometido a necropsia en una clínica privada de la ciudad de Villarrica. Fueron inspeccionados la piel y anexos, para luego realizar una incisión ventral abarcando tórax y abdomen en donde fueron exploradas las cavidades y órganos, registrándose la presencia macroscópica de lesiones y parásitos.

Se obtuvieron 3 muestras de aproximadamente 50 gramos cada una, de músculo intercostal, maseteros y diafragma para examen fototriquinoscópico por compresión, se efectuaron 10 cortes de cada grupo muscular, los cuales fueron comprimidos entre dos placas de triquinoscopía y observadas en aumento total de 40X. Las muestras de músculo con larvas enquistadas observadas fueron conservadas en lactofenol. Posteriormente, se realizó digestión artificial de 20 gramos totales, para la obtención de larvas de Trichinella, en el Laboratorio de Parasitología de la Universidad Santo Tomás, sede Temuco, utilizando una solución de pepsina-ácido clorhídrico al 1\% en proporción muestra-solución de 1:15. La muestra fue macerada, mezclada con la solución y agitada de forma constante por agitador magnético a $45^{\circ} \mathrm{C}$ por 30 minutos y recuperadas mediante embudo de decantación. Las larvas obtenidas y el resto del músculo fueron derivados al Laboratorio de Inmunoparasitología Molecular de la Universidad de La Frontera para realizar infección experimental en ratones mediante carnivorismo.

\section{RESULTADOS Y DISCUSIÓN}

El ejemplar de Puma concolor, al examen macroscópico presentó deshidratación y emaciación severa, ausencia de contenido estomacal, hígado de aspecto pálido, sugerente de infiltración grasa y lesiones lacerantes en extremidades y cola, caracterizado como ataque por otro congénere. La data de muerte estimada en base al registro de la señal de mortalidad, fue de aproximadamente tres días.

No fueron observados ectoparásitos y sólo fueron recuperados del intestino delgado escasos ejemplares de cestodos altamente deteriorados.

En los 10 cortes realizados en diafragma e intercostales, se identificó al menos un quiste por corte. La carga parasitaria del ejemplar fue de 5 larvas por gramo de músculo, obtenida de la digestión artificial, la cual es baja si comparamos con infecciones naturales en cerdos y ratas (Alcaíno y col 1981, Schenone y col 1967º , pero similar con aquella observada por Ribicich y col (2010) en un puma argentino. La infección practicada en ratas de laboratorio no tuvo resultado, lo cual ha limitado la disponibilidad de material de estudio. Al respecto, Nelson y Mukundi (1963) postulan que cepas aisladas de algunos carnívoros silvestres presentan, en ocasiones, una reducida infectividad para las ratas en condiciones experimentales.

La presencia de Trichinella sp. en animales silvestres mantiene la transmisión de la infección en la naturaleza, constituyendo un potencial riesgo para la salud pública (Soulsby 1987). En Argentina, Ribicich y col (2005, 2010) han descrito la infección en animales introducidos y nativos como jabalí (Sus scrofa), ratas asilvestradas (Rattus norvegicus), quirquincho (Chaetophractus villosus) y puma (Puma concolor), incluyendo diversos casos de infección humana por consumo de carne de puma, con uno de ellos documentado en la Patagonia (Mansilla y col 1994). En Chile, estudios realizados en animales silvestres nativos no han demostrado la presencia del parásito (Alcaíno y Arenas 1981), la única evidencia de trichine- 
llosis humana adquirida desde el medio natural, ha sido descrita por consumo de jabalí (Sus scrofa) especie silvestre introducida en Chile (García y col 2005).

La infección por Trichinella en $P$. concolor puma puede atribuirse a la variedad de presas hospedadoras, entre estas, roedores silvestres y sinantrópicos, como $R$. norvegicus. Estudios de dieta de pumas basados en el análisis de fecas lo relacionan al consumo de jabalí, roedores y carnívoros menores de hábitos depredadores o carroñeros (Pessino y col 2001, Pacheco y col 2004), todos ellos potenciales hospedadores del parásito.

El hallazgo de este puma infectado por Trichinella sp. en la Región de La Araucanía, representa un registro valioso sobre la presencia del parásito en animales nativos de Chile, constituyendo una contribución al conocimiento de esta parasitosis escasamente demostrada en el medio silvestre.

\section{REFERENCIAS}

Acha PN, B Szyfres. 2001. Zoonosis y enfermedades transmisibles comunes al hombre y a los animales. Volumen III: Parasitosis. $3^{\mathrm{a}}$ ed. OPS Publicación Científica y Técnica, Washington D.C., EEUU, Pp 544.

Alcaíno H, X Arenas. 1981. Antecedentes sobre triquinosis en Chile. Monografías de Medicina Veterinaria 3, 29-42.

Alcaíno H, X Arenas, A Puig, J Plaza, G Sánchez, X Brito. 1981. Triquinelosis en cerdos autorizados legalmente para el consumo. Rev Med Chile 109, 516-518.

Fonseca-Salamanca F, JJ Nogal-Ruiz, C Benito, MV Camacho, AR Martínez-Fernández. 2006. Molecular characterization of Trichinella genotypes by inter simple sequence repeat polymerase chain reaction (ISSR-PCR). J Parasitol 92, 606-610.

García E, L Mora, P Torres, M Jercic, R Mercado. 2005. First record of human trichinosis in Chile associated with consumption of wild boar (Sus scrofa). Mem Inst Oswaldo Cruz 100, 17-18.

Krivokapich SJ, CL Prous, GM Gatti, V Confalonieri, V Molina, H Matarasso, E Guarnera. 2008. Molecular evidence for a novel encapsulated genotype of Trichinella from Patagonia, Argentina. Vet Parasitol 156, 234-240.

Lardiez M. 1964. Prevalencia de triquinosis en perros y gatos de la ciudad de Santiago. Tesis Licenciatura Medicina Veterinaria, Facultad de Ciencias Veterinarias, Universidad de Chile, Santiago, Chile.

La Rosa G, G Marucci, E Pozio. 2003. Biochemical analysis of encapsulated and non-encapsulated species of Trichinella (Nematoda, Trichinellidae) from cold- and warmblooded animals reveals a high genetic divergence in the genus. Parasitol Res 91, 462-466.

Laundre JW, L Hernández, D Streubel, K Altendorf, C López González. 2000. Aging mountain lions using gum-line recession. Wildl Soc Bull 28, 963-966.

Letonja T, S Ernst. 1974. Triquinosis en perros de Santiago, Chile. Bol Chil Parasitol 29, 51.

Mansilla R, H Soloaga, R Guillén, C Segura. 1994. Reporte de dos casos clínicos de triquinosis en la Patagonia Austral, a partir de la ingesta de carne de puma infectada. Resúme- nes del II Congreso Argentino de Zoonosis, Buenos Aires, Argentina, Pp 179.

Martínez AR. 1999. Triquinelosis. En: Cordero del Campillo M, Rojo F (eds). Parasitología Veterinaria. McGraw-HillInteramericana, Madrid, España, Pp 496-497.

Murrell KD, JR Lichtenfelsd, S Zarlenga, E Pozio. 2000. The systematic of the genus Trichinella with a key to species. Vet Parasitol 93, 293-307.

Neghme A, G Hoecker, E Felner. 1948. Incidencia de triquinosis en las ratas de basural y su posible relación con el grado de infestación de los cerdos criados en el mismo sitio. Rev Chil Hig Med Prev 10, 209-212.

Neghme A, H Schenone. 1970. Trichinosis in Latin America. In: Gould SE (ed). Trichinosis in Man and Animals. Charles C Thomas, Illinois, USA, Pp 407-422.

Nelson GS., J Mukundi. 1963. A strain of Trichinella spiralis from Kenya of low infectivity to rats and domestic pigs. $J$ Helminth 37, 329-338

Oberg C, S Ernst, P Linfati, P Martin. 1979. Triquinosis en perros de la comuna de Máfil, Provincia de Valdivia, Chile. Bol Chil Parasitol 34, 46-47.

Pacheco L, A Lucero, M Villca. 2004. Dieta de Puma (Puma concolor) en Parque Nacional Sajama, Bolivia y su conflicto con la ganadería. Ecol Boliv 39, 75-83.

Pessino M, J Sarasola, C Wander, N Besoky. 2001. Respuesta a largo plazo del puma (Puma concolor) a una declinación poblacional de la vizcacha (Langostomum maximus) en el desierto de El Monte, Argentina. Ecol Austral 11, 61-67.

Plaza J, H González, L Pinochet. 1961. Decomisos por cisticercosis y triquinosis efectuados en el Matadero Industrial de San Miguel. Libro 40 Conv Nac Med Vet de Chile, Pp 155-158.

Pozio E. 2000. Factors affecting the flow among domestic, synanthropic and sylvatic cycles of Trichinella. Vet Parasitol 93, 241-262.

Pozio E, E Hoberg, G La Rosa, D Zarlenga. 2009. Molecular taxonomy, phylogeny and biogeography of nematodes belonging to the Trichinella genus. Infect Genet Evol 9, 606-616.

Quintana V, J Yáñez, M Valdebenito. 2000. Orden Carnívora. En: Muñoz-Pedreros A, Yáñez J. (eds). Mamíferos de Chile. CEA, Valdivia, Chile, Pp 155-187.

Ribicich M, HR Gamble, A Rosa, J Bolpe, A Franco. 2005. Trichinellosis in Argentina: an historical review. Vet Parasitol 132, 137-142.

Ribicich M, H Gamble, J Bolpe, E Scialfa, S Krivokapich, N Cardillo, A Betty, M Cambiaggi, M Pasqualetti, F Fariña, A Rosa. 2010. Trichinella infection in wild animals from endemic regions of Argentina. Parasitol Res 107, 377-380.

Rodríguez H, I Noemí, JL Cerva, O Espinoza-Navarro, ME Castro, M Castro. 2011. Análisis paleoparasitológico de la musculatura esquelética de la momia del cerro El Plomo, Chile: Trichinella sp. Chungará, Revista de Antropología Chilena, 43, 581-588.

Rojas A, F Villarroel, F Díaz, P Rubio, H Schenone.1971. Investigación de triquinosis y capilariasis hepática en Rattus norvegicus del Matadero Municipal de Santiago (Chile). Bol Chil Parasitol 26, 65-66.

SAG, Servicio Agrícola y Ganadero del Ministerio de Agricultura Chile. 2010. La ley de caza y su reglamento. $12^{\mathrm{a}}$ ed. Unidad Comunicación y Prensa SAG, Santiago, Chile, Pp 30-50.

Sanhueza C, M Álvarez. 1980. Zoonosis comprobadas en animales beneficiados en los mataderos del país durante 
1979. Bol Vigilancia Epidemiológica. Chile. Ministerio Salud 7, 2-4.

Schenone H, J Carrasco, F Villarroel, R Chinchón, J Urriola. 1967ª. Epizootias de triquinosis porcina y su asociación con desratizaciones con Warfarin. Bol Chil Parasitol 22, 138-143.

Schenone H, C Jacob, A Rojas, F Villarroel. 1967 ${ }^{\text {. Infección }}$ por Trichinella spiralis en Rattus norvegicus capturados en el Matadero Municipal de Santiago de Chile. Bol Chil Parasitol 22, 176.

Schenone FH, A Olea, H Schenone C, L Contreras, R Mercado, L Sandoval, C Pavletic. 2002. Situación epidemiológica actual de la triquinosis en Chile 1991-2000. Rev Med Chile 130, 281-285.

Shaw HG, P Beier, M Culver, M Grigione. 2007. Puma Field Guide. The Cougar Network, Concord, MA, USA.

Soulsby E. 1987. Parasitología y enfermedades parasitarias en los animales domésticos. 7 ed. Nueva Editorial Interamericana, México D.F., México, Pp 332-333.

Wilson D, DA Reeder. 2005. In: Wilson D, Reeder D (eds). Mammal Species of the World: A Taxonomic and Geographic Reference. $3^{\text {rd }}$. The John Hopkins University Press, Baltimore, Maryland, USA, Pp 2142. 